IFUP-TH 2002/31, TRINLAT-02/03

\title{
A Hamiltonian lattice study of the two-dimensional Wess-Zumino model
}

\author{
Matteo Beccaria ${ }^{\mathrm{a}}$, Massimo Campostrini $^{\mathrm{b} *}$ and Alessandra Feo ${ }^{\mathrm{c}}$ \\ a Dipartimento di Fisica dell'Università di Lecce and I.N.F.N., Sezione di Lecce \\ bDipartimento di Fisica "Enrico Fermi" dell’Università di Pisa and I.N.F.N., Sezione di Pisa \\ ${ }^{\mathrm{c}}$ School of Mathematics, Trinity College, Dublin 2
}

We investigate a Hamiltonian lattice version of the two-dimensional Wess-Zumino model by Quantum Monte Carlo simulations. In order to study the pattern of supersymmetry breaking, we measure the ground state energy and the correlation length along a trajectory approaching the continuum limit. The algorithm is very effective in measuring the ground state energy, and adequate for the correlation length.

\section{INTRODUCTION}

Numerical simulations of lattice field theories are usually performed in the Lagrangian formulation. Nonetheless, we think there are very good reasons to develop numerical simulation techniques for the Hamiltonian approach [1] : powerful many-body techniques are available [2], which allow the direct computation of the vacuum wave function properties; fermions are implemented directly and need not be integrated out; properties like the mass spectrum are more immediate. Finally, universality checks between the Lagrangian and the Hamiltonian formalism are very welcome.

\section{THE MODEL}

We study the Hamiltonian lattice version of the two-dimensional Wess-Zumino model described in Refs. [3, [4]; we only wish to highlight here the main features of the formulation.

In the Hamiltonian formalism, since $H$ is conserved, it is possible to preserve exactly a 1dimensional subalgebra of the original supersymmetry algebra, i.e., we can write $H=Q^{2}$, where $Q$ is a fermionic charge. This subalgebra is enough to guarantee some of the most important property of supersymmetry, including a nonnegative spectrum, and pairing of fermionic and bosonic states of nonzero energy; spontaneous breaking of supersymmetry is equivalent to a

\footnotetext{
*Presented by M. Campostrini
}

strictly positive ground-state energy $E_{0}$; the full supersymmetry algebra is recovered in the continuum limit together with Lorentz invariance.

In order to obtain a Hamiltonian free of fermion sign problems, and therefore amenable to Quantum Monte Carlo methods, we adopt free boundary conditions, with lattice size $L \equiv 2(\bmod 4)$.

The model is parametrized by a prepotential $V(\phi)$, an arbitrary polynomial in the bosonic field. The two-dimensional Wess-Zumino model is superrenormalizable; fields do not renormalize, and only $V(\phi)$ needs to be normal ordered.

In strong coupling at leading order, the model reduces to independent copies of supersymmetric quantum mechanics, one for each site; supersymmetry is broken if and only if the degree of the prepotential $V$ is even [5]. In weak coupling, on the other hand, supersymmetry is broken at tree level if and only if $V$ has no zeroes. The predictions of strong coupling and weak coupling are quite different, and it is interesting to study the crossover from strong to weak coupling.

\section{MONTE CARLO SIMULATIONS}

We perform our simulations using the Green Function Monte Carlo (GFMC) algorithm [2]. A discussion of GFMC in the context of the present problem can be found in Ref. [4]; we only wish to remark the main features of the algorithm: the aim is to generate a stochastic representation of the ground-state wavefunction, which is then 
used to compute expectation values of observables. Statistical fluctuations are reduced with the help of a guiding wavefunction, whose free parameters are determined dynamically during the simulation. In order to keep the variance of observables finite as the simulation proceeds, it is necessary to simulate a population of $K$ walkers (field configurations at fixed time), and extrapolate the results to $K \rightarrow \infty$.

We focus on the case $V=\lambda_{2} \phi^{2}+\lambda_{0} \phi$; strong coupling always predicts supersymmetry breaking; weak coupling predicts unbroken supersymmetry for $\lambda_{0}<0$; according to Ref. [6], unbroken supersymmetry should be accompanied by a nonzero $\langle\phi\rangle$ (parity breaking).

We presented preliminary results, obtained by GFMC, for intermediate couplings in Ref. [3]; our aim is to extend the study towards the continuum limit and to larger lattices.

Perturbative computations show that

$\lambda_{2} \approx a \lambda_{2}^{\mathrm{ren}}, \quad \lambda_{0} \approx a \lambda_{0}^{\mathrm{ren}}+a \lambda_{2}^{\mathrm{ren}} \frac{1}{2 \pi} \ln (a M)$,

where $\lambda_{i}$ is the adimensional lattice bare coupling, $\lambda_{i}^{\text {ren }}$ is the renormalized (continuum) coupling, with dimension of $m^{1}$, defined at the mass scale $M$, and $a$ is the lattice spacing. We study, as $\lambda_{2} \rightarrow 0$, the trajectory

$\lambda_{0}=\frac{\lambda_{2}}{2 \pi} \ln \left(4 \lambda_{2}\right)$,

corresponding to a perturbative RG trajectory (11); the effect of $\lambda_{0}$ is small in the range we considered, therefore we expect Eq. (22) to be a reasonable approximation to a true RG trajectory.

We estimate the correlation length from the exponential decay of the connected correlation function $G_{d}=\left\langle\phi_{n} \phi_{m}\right\rangle_{c}$ averaged over all $n, m$ pairs with $|m-n|=d$, excluding pairs for which $m$ or $n$ is closer to the border than (typically) 8 . In our formulation, fermions are staggered and even/odd $d$ correspond to different channels.

We begin with the discussion of the case $V=$ $0.35 \phi^{2}+0.02$, for which we have obtained the statistics of $4 \times 10^{6}$ GFMC iterations. The even- $d$

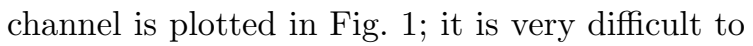
extract a correlation length, presumably because $\phi$ has a very small overlap with the lightest state

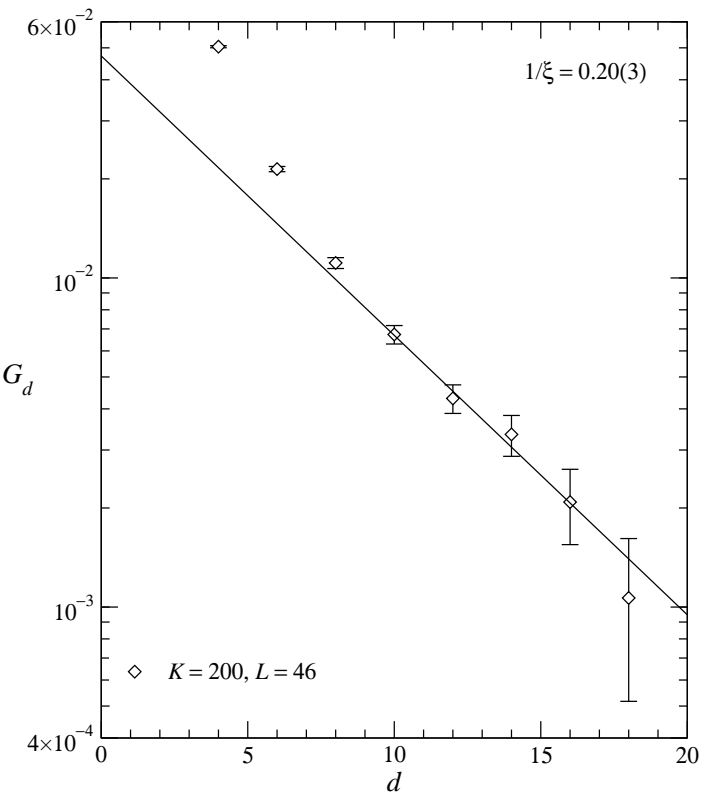

Figure 1. Connected correlation of $\phi$ at even distance for $V=0.35 \phi^{2}+0.02$; the solid line is an exponential fit from distance 8 to $18 ; L=34$ and $K=100$ data are consistent with the data shown.

of the channel, and the value of $\xi$ quoted in Fig. 1 should be considered tentative. The odd- $d$ channel, plotted in Fig. 2, is much cleaner, and it is possible to estimate $\xi$ with a good precision.

For the other values of $\lambda_{2}$, the situation is similar but with larger errors; we have a statistics of at least $10^{6}$ iterations, which we are increasing to $4 \times 10^{6}$. The values of $\xi_{\text {odd }}$ follow nicely the expected behavior $\xi \propto 1 / \lambda_{2}$, as shown in Fig. 3: the entire range $0.088 \leq \lambda_{2} \leq 0.35$ seem to be in the scaling region, with $\lambda_{2}=0.5$ a borderline case. The values of $\xi_{\text {even }}$ have very large errors, and it is hard to draw any conclusion from them.

We measure the ground state energy $E_{0}$ along the trajectory (2); the measurements have a very small statistical error, ranging from $1 \%$ for $\lambda_{2}=$ 0.044 (where $E_{0} / L \approx 10^{-3}$ ) to $0.1 \%$ for $\lambda_{2}=0.5$. We extrapolate to $L \rightarrow \infty$ and $K \rightarrow \infty$ fitting $E_{0} / L$ to the form

$E_{0} / L=\mathcal{E}_{0}\left(1+c_{L} / L+c_{K} / K\right)$.

$E_{0} / L$ is plotted in Fig. 国: it seems to behave 


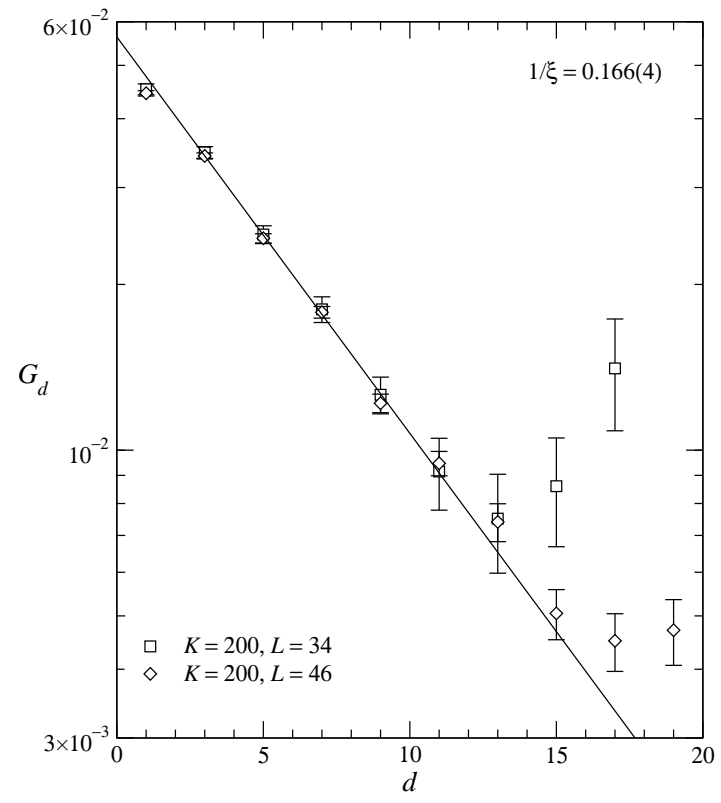

Figure 2. Connected correlation of $\phi$ at odd distance for $V=0.35 \phi^{2}+0.02$; the solid line is an exponential fit from distance 3 to $15 ; K=100$ data are consistent with the $K=200$ data shown.

$\propto \lambda_{2}^{5 / 3}$, while naïve scaling would predict $\propto \lambda_{2}^{2}$. The value of $E_{0} / L$ (disregarding this puzzling exponent) and the lack of any signal for a breakdown of parity (like a double-peaked distribution of $\phi$ ) strongly hint that the trajectory (2) belongs to the phase with broken supersymmetry and zero $\langle\phi\rangle$. We are repeating the computation for trajectories with smaller $\lambda_{0}$.

\section{REFERENCES}

1. J. Kogut, L. I. Susskind, Phys. Rev. D11 (1975) 395; J. Kogut, Rev. Mod. Phys. 51 (1979) 659.

2. W. von der Linden, Phys. Rept. 220 (1992) 53.

3. M. Beccaria, M. Campostrini, A. Feo, Nucl. Phys. B (Proc. Suppl.) 106-107 (2002) 944.

4. M. Beccaria, M. Campostrini, A. Feo, heplat/0109005.

5. E. Witten, Nucl. Phys. B188 (1981) 513.

6. E. Witten, Nucl. Phys. B202 (1982) 253.

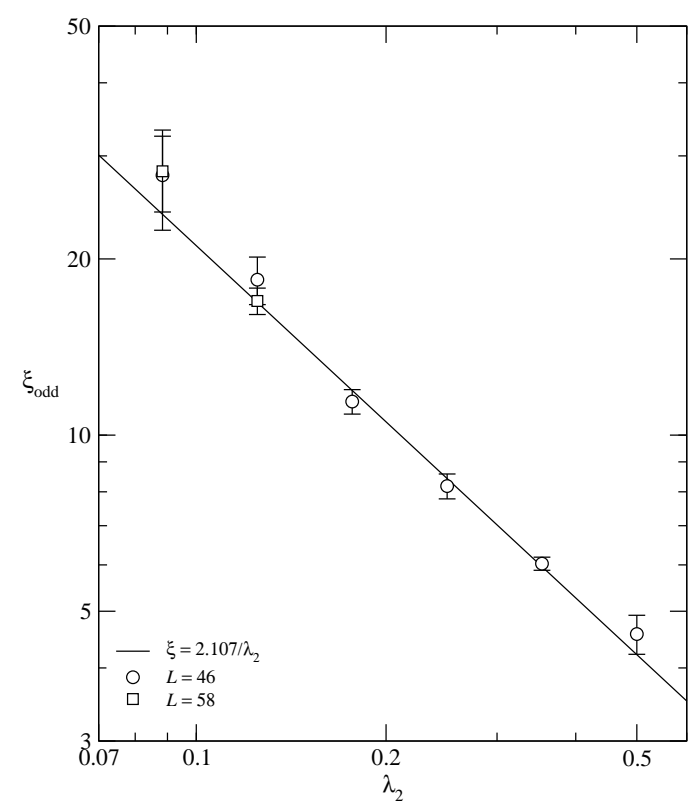

Figure 3. Correlation length in the odd distance channel vs. $\lambda_{2}$; the solid line is a scaling fit.

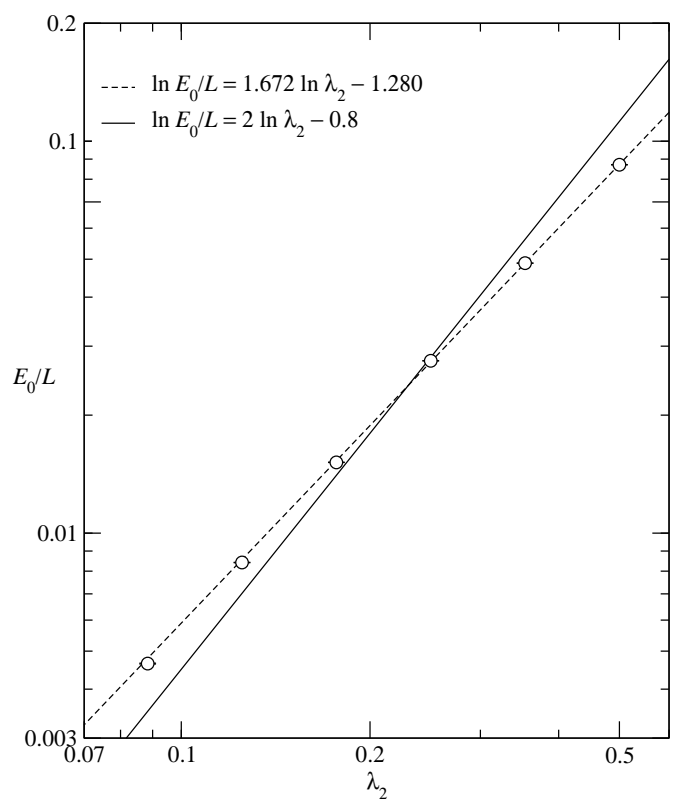

Figure 4. Ground-state energy density vs. $\lambda_{2}$. The dashed line is the result of a fit; the solid line shows the naïve scaling behavior. 